\section{Gene therapy in Anderson- Fabry disease. State of the art and future perspectives}

\author{
Giorgio Spiniello, Federica Verrillo, \\ Riccardo Ricciolino, Dario Prozzo, \\ Andrea Tuccillo, Martina Caiazza, \\ Marta Rubino
}

Department of Cardiothoracic Sciences, Università degli Studi della Campania Luigi Vanvitelli, Naples, Italy

\begin{abstract}
Anderson-Fabry disease (AFD) is an Xlinked lysosomal storage disorder caused by a deficiency of the lysosomal enzyme, agalactosidase $\mathrm{A}$. The inadequate enzymatic activity leads to systemic storage of glycosphingolipids, mostly globotriaosylceramide, in the lysosomes. As of now, enzyme replacement therapy is the only approved treatment for AFD. However, it does not induce a complete and lasting response in several clinical contexts. Genemediated enzyme replacement is an emerging approach that could overcome these limits. The single gene nature of AFD enhances the possibility to transfect and modify a small number of cells, making them capable to affect the correction of a larger number of cells. This review summarizes the history and the state of the art of gene therapy in AFD, showing potential benefits and limits.
\end{abstract}

\section{Gene therapy in Anderson-Fabry disease}

Anderson-Fabry disease (AFD) is a Xlinked storage disorder caused by a lysosomal enzymatic deficiency. The $\alpha$-galactosidase A (GLA) mutations lead to accumulation of several substrates involved in glycosphingolipids metabolism like Globotriaosylsphingosine (Gb3). ${ }^{1}$ The lysosomal storage of $\mathrm{Gb} 3$ appears to be the main cause of organ failure in Fabry disease, involving most frequently kidney, brain and heart. Common symptoms are angiokeratomas, acroparesthesia, neuropathic pain, cerebrovascular disease, renal failure, cardiomyopathy. ${ }^{2}$

AFD prevalence is estimated to range from $1: 8454$ to $1: 117,000$ males, ${ }^{3}$ while worldwide incidence of AFD is reported to be in the range of 1 in 40,000-117,000.

Even though Fabry disease is an $\mathrm{X}$ linked pathology, it does not involve only men. Women carriers can be affected, developing a mild, late onset disease due to the random inactivation (lyonization) of Xchromosome that results in a heterogeneous enzyme expression in every cell. ${ }^{5}$ Based on the lyonization, some organs in female carriers will have low GLA expression and will develop the disease and others will have normal GLA expression. Because of the random X-chromosome's genes expression, about two-thirds of women carriers will develop the disease.

The GLA gene is situated in the long arm of the X-chromosome. It originally consists of $12 \mathrm{~kb}$ with 7 exons, encoding for a 370 amminoacids-protein after the splicing process. There are nearly a thousand identified mutations in the GLA gene implied in AFD, including missense, nonsense, small deletions, small insertions, splice defects and rearrangements. ${ }^{6}$ The wide majority are missense mutations with a single amminoacid substitution in the GLA gene. Despite this, there are a lot of mutations of unknown significance.

Cardiovascular imaging like echocardiogram and cardiovascular magnetic resonance (CMR) can provide important information for the correct diagnosis of cardiovascular involvement in Fabry disease. The most relevant feature on echocardiogram is the left ventricular hypertrophy $(\mathrm{LVH})$ with normal ejection fraction, similar to hypertrophic cardiomyopathy (HCM). ${ }^{7} \mathrm{CMR}$ can play a central role in differentiating AFD from HCM and can detect early cardiac involvement, assessing ventricular function and tissue characterization by means of late gadolinium enhancement (LGE) and T1 mapping. ${ }^{8}$ The diagnostic assessment for AFD is beyond the scope of this review.

Definitive diagnosis is based on dosage of plasma enzyme activity, histologic evidence of intracellular Gb3 accumulation and genetic testing with the detection of the pathological mutations. High plasma levels of $\mathrm{Gb} 3$ and Lyso-Gb3 represent sensitive biomarkers. The reducing of at least one third of the normal value of GLA activity is considered pathogenetic. However, lots of studies show the necessity to evaluate biological, histological and clinical alterations without a validated threshold. ${ }^{9}$ Low enzyme activity in plasma is often sufficient in affected males for a definitive diagnosis, whilst in women it is mandatory a genetic confirmation, because of the lyonization phenomenon. Once the diagnosis has been achieved, screening of all members of the family is recommended.

Heart involvement is one of the main complications and it emerges in adulthood, but early heart damage can be observed in young patients, like valvular disfunction,
Correspondence: Marta Rubino, Department of Cardiothoracic Sciences, Università della Campania Luigi Vanvitelli, Ospedale MedicoChirurgico AORN Monaldi, Naples, Italy.

E-mail: rubinomarta@libero.it

Key words: Cardiomyopathies; AndersonFabry disease; gene therapy.

Contributions: Over time all authors listed in the manuscript have substantially contributed to it. All Authors have read and approved the final version of the manuscript.

Conflict of interests: the authors declare no conflict of interests.

Received for publication: 2 May 2020.

Revision received: 25 June 2020

Accepted for publication: 20 July 2020.

This work is licensed under a Creative Commons Attribution NonCommercial 4.0 License (CC BY-NC 4.0).

(C) Copyright: the Author(s), 2020

Licensee PAGEPress, Italy

Cardiogenetics 2020; 10:9075

doi:10.4081/cardiogenetics.2020.9075

conduction abnormalities and mild LVH. Histologic appearance is typical, with a binary appearance of left ventricular endocardial border. Most of the cardiac complications emerge in adulthood, but early progressive heart damage can be observed in young patients. For this reason, ECG-Holter monitoring and a comprehensive echocardiographic and electrocardiographic evaluation is suggested during every clinical assessment.

However, the deposit of Gb3 leads to multi-organ disease. Renal impairment is often a major concern in AFD patients. The accumulation of Gb3 may occur prenatally in renal cells before any measurable abnormality in routine tests or GFR declination and albuminuria is usually the earliest pathological sign. Cerebrovascular events (stroke and transient ischemic attacks) are extremely common in adult AFD patients as they represent the second leading cause of death. ${ }^{10}$ The underlying mechanism is the increased release of ROS due to Gp3 accumulation with consequent inflammatory cells activation. AFD patients also suffer from neuropathic pain due to degeneration of myelinated A-delta fibers (acroparesthesias). It primarily affects feet and hands but can later progress proximally. ${ }^{11}$ Abdominal pain is a very common symptom in AFD patients and may mimic inflammatory bowel disease, sometimes requiring endoscopic analyses for the differential diagno- 
sis. Typical and recognizable lesions are also found on the skin (angiokeratomas) and the eyes (cornea verticillata) and they can be a red flag in the diagnostic evaluation of a patient, increasing the probability of AFD.

Considering the aforementioned clinical characteristics of AFD patients, the disease should be suspected in males or females with a combination of the following clinical features: Intermittent episodes of burning pain in the extremities (acroparesthesias); cornea verticillata; abdominal pain; nausea, and/or diarrhea of unknown etiology in young adults; arrhythmias of unknown etiology, particularly in young adults; cutaneous vascular lesions (angiokeratomas); diminished perspiration (hypoor anhidrosis); stroke of unknown etiology; chronic kidney disease (CKD) and/or proteinuria of unknown etiology, especially if associated with multiple renal sinus cysts discovered incidentally; LVH of unknown etiology.

AFD therapy was previously based on symptomatic drugs like analgesics, diuretics and angiotensin converting enzyme inhibitors (ACE-I) ${ }^{12}$ and life expectancy was low, mainly influenced by cardiac involvement, with arrhythmias being the most common cause of death. ${ }^{13}$

The introduction of enzyme replacement therapy (ERT) in the early 2000's has dramatically changed the outcome of AFD patients. The correct timing for ERT initiation is of mandatory importance. ERT should be considered in boys older than 8 years with classical form even if asymptomatic. Instead, AFD patients with non-classic, attenuated and late onset variants should be monitored closely and treated once symptoms appear or when there is renal biopsy evidence of disease. ${ }^{14}$ In fact, in classical Fabry mutations there is histological evidence of Gb3 accumulation with cellular and vascular injury in renal tissue in absence of proteinuria or other significant clinical evidences. ERT is based on the use of two recombinant GLA enzyme preparations, agalsidase alfa and agalsidase beta, produced in a cultured human cell line. They are both available in Europe. ERT leads to a not worsening or in stabilization of the disease and sometimes improves kidney and heart function, especially if it is started in an early phase. ${ }^{15}$ However, ERT is not defects-free. In fact, it does not achieve satisfying results when target organs are severely damaged. ${ }^{16}$ ERT can cause, in about $40 \%$ of all ERT-treated males, the production of neutralizing antidrug antibodies (ADAs) that limit the efficacy of the therapy. ${ }^{17}$ Another cause of unsatisfactory results is represented by an inadequate brain and bones drug penetration, ${ }^{18}$ and this is unfortunate because stroke is one of the most significant manifestations in AFD patients and represents an important cause of premature death. ERT is usually well tolerated, with no major side effects directly related to it aside from transient infusionassociated reactions (IARs). IARs are probably the result of anaphylactoid reactions (compound-mediated) and not anaphylactic (IgE-mediated type 1 hypersensitivity). ${ }^{18}$

Because of ERT limits, new therapeutic strategies are being studied and tested, including stem cells therapy, chaperones therapy and gene therapy.

Pharmacological chaperones are molecules that act binding the mutant GLA enzyme, improving its stability and favoring its transfer to the lysosomes ${ }^{19}$ (Figure 1). Unfortunately, this therapy is very effective only in non-classical phenotypes, that are characterized by missense mutations and are thereby more sensible to this chaperoning effect. At the moment, the compound DGJ (Migalastat) is currently in Phase III clinical trial, after positive results in phase II for safety and tolerance, and the results are encouraging. Migalastat is a low molecular weight analogue of the terminal galactose residue on GL-3 that binds selectively and reversibly to the active sites of amenable mutant forms of $\alpha$-galactosidase

A enzyme..$^{20}$ Once in lysosomes, migalastat dissociates from $\alpha$-galactosidase A allowing the enzyme to break down GL-3. After dissociation from the enzyme, Migalastat is rapidly removed from the cell and excreted. The pharmacokinetics of migalastat are not altered to a clinically relevant extent by gender or race. The use of Migalastat has not been studied in patients with Fabry disease who have severe renal impairment, or who have hepatic impairment; however, hepatic impairment is not expected to affect the pharmacokinetics of migalastat, based on the metabolism and excretion pathways. Two randomized and multicenter phase 3 trials are in progress to prove the efficacy of Migalastat: FACETS (a placebo controlledtrial) and ATTRACT (active comparator controlled trial). In phase 3 trials, eligible patients were required to have a migalastatamenable GLA mutation based on the initial HEK-293 assay. ${ }^{21}$ Migalastat is well tolerated and mostly adverse events (AEs) are mild. Most common AEs are headache, infusion-associated reactions, nasopharyngitis, urinary tract infection and nausea. Because of its effectiveness and the possibility of oral administration, Migalastat will probably be one of the most important options for AFD treatment. The limitations of this approach are its effectiveness limited to AFD patients with missense mutations

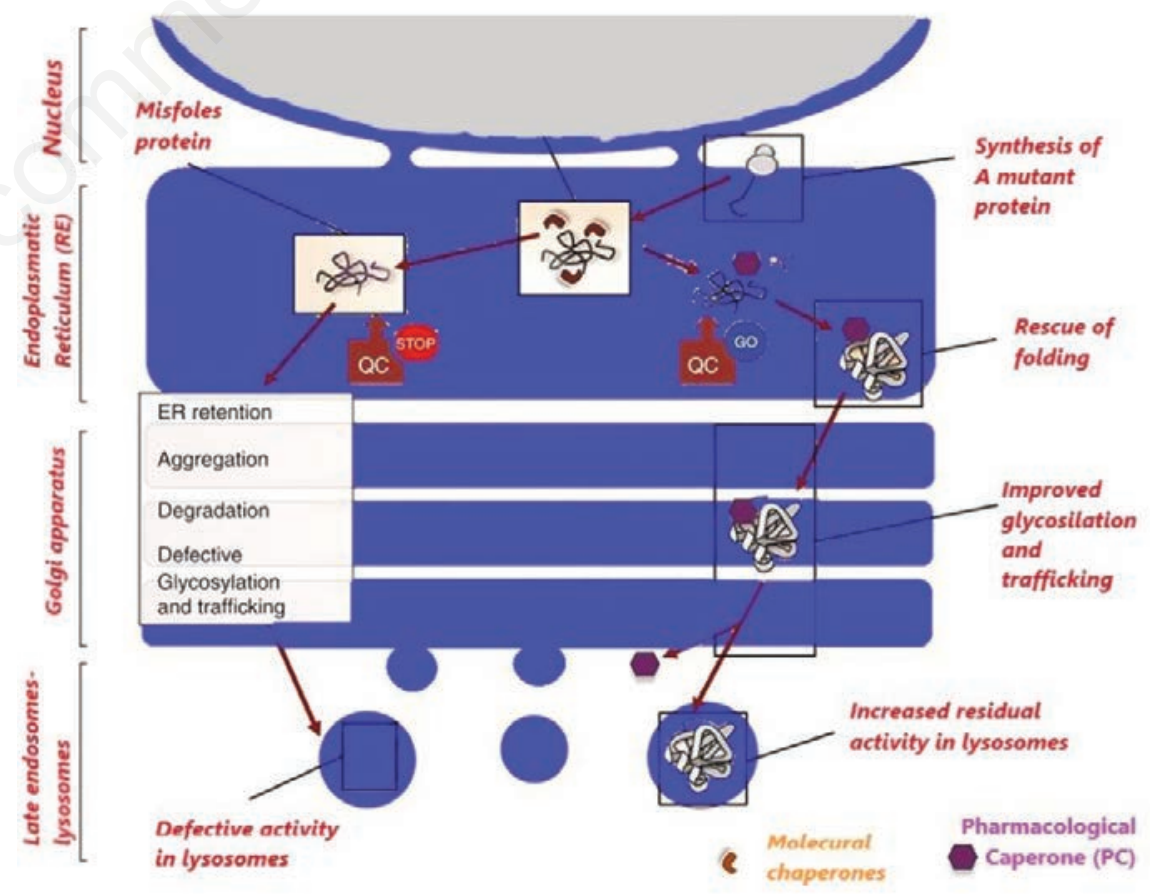

Figure 1. Mechanism of action of pharmacological chaperones. Pharmacological chaperones are molecules that act binding the mutant $\alpha$-galactosidase $A$ enzyme, improving its stability and favoring its transfer to the lysosomes thus increasing residual activity in lysosomes. 
causing expression of misfolded proteins and the major immunogenicity and infusion-associated reactions compared to ERT. ${ }^{22}$ Better results are likely to be obtained by combining multiple approaches, as already demonstrated with chaperons plus ERT therapy. ${ }^{23}$

An important disease mechanism that has been recognized and identified is the pseudoexon activation. These are instable portions of DNA that added to a single nucleotide variation could break the equilibrium between positive and negative splicing regulatory factors, modifying disease expression. ${ }^{24}$ The splice switching oligonucleotides (SSO) has been proposed as an alternative therapy based on the aberrant splicing mechanism. ${ }^{25} \mathrm{SSO}$ acts recovering the correct splicing pattern and closing communication opportunities between splicing regulatory elements (SRE), splicesoma components and their binding sequences, restoring the expression of wildtype mRNA. This therapy has been studied in patients with $\mathrm{C} 936+919 \mathrm{G}>\mathrm{A}$, a mutation that causes a premature TGA stop codon and consequently a truncated protein. Fortunately, SSO reach high concentrations in heart and kidney that are organs usually affected in patients with this mutation.

A novel approach in AFD treatment is gene therapy, with the aim of introducing a healthy copy of the GLA gene to restore normal levels of the alpha-galactosidase A enzyme. It mainly consists in gene transference that can be obtained ex-vivo and/or invivo. In vivo strategy consists in systemic or local administration of viral vectors carrying GLA gene, while in ex vivo strategy cultures of extracted patient stem cells are transfected using virus vectors in vitro and then reimplanted. ${ }^{26}$

Vectors used for in vivo strategy are modified viruses like adenovirus or lentivirus (Figure 2). The use of this kind of therapy could lead to long term therapeutic effects, absence of adverse effects and, moreover, it appears to be more cost-effectiveness than ERT. Promising results have already been achieved in animal models, such as mice models. ${ }^{27}$ Several factors appear to be related to the therapeutic effect (sex of mice, type of vectors and method of administration).

Studies report a $20-35 \%$ GLA activity and $\mathrm{Gb} 3$ reduction in the livers of mice transfected with modified adenovirus via hepatic portal vein. No notable immune response was recorded in the transfected animals. ${ }^{28}$ The main disadvantage was portal vein delivery and the need of multiple injections. Another group tested a new route of administration via intramuscular injection of the AAV (adeno-associated viruses, non-enveloped single-stranded DNA viruses), reporting a complete elimination of Gb3 from body tissues. ${ }^{29}$

Another strategy in study is the administration of recombinant $\mathrm{AAV}$ driven by modified chicken beta actin promoter and CMV promoter, trying to improve the enzyme level of expression. In this context the newest strategy is characterized by AAV vector conjoint to a liver specific promoter. Two copies of a human prothrombin enhancer linked to the human serum albumin promoter (Hprt(2)HAS) are used. ${ }^{30}$ In mice subjected to this treatment, investigators found higher levels of GLA expression than other promoters. To improve transduction efficiency, pseudo-typed AAV2 and AAV2/5 vectors have been created to encode the missing lysosomal enzyme.

The use of other retroviral vectors, particularly Lentivirus, has also been investigated. ${ }^{31}$ Due to their capacity to infect nondividing cells thanks to the expression of viral accessory proteins and nuclear localization signals and the excellent tissue trophism, lentivirus attracted the interest of many scientists.

Human tests started in 2017 with encouraging results, and an open label phase one clinical trial (NCT02800070) is currently ongoing in Canada, while another experimental gene therapy (NCT03454893) is about to start the phase one study. ${ }^{32}$ They are both based on the use of a lentivirus vector, infecting the $\mathrm{Cd} 34+$ stem cells obtained from the patient, enabling them to express GLA. An autologous cell transplant is then performed. A small number of transduced cells can affect the correction of a larger number of non-transduced cells in vivo (cross-correction). ${ }^{33}$

These studies are focusing on 18 to 50 years old male patients with AFD defined by very low or absent $\alpha$-gal A activity and a classic phenotype who were on ERT prior to enrolment.

Despite the advantages they offer, virus vectors can induce inflammatory responses, possibly stimulating antibodies production and consequent decrease of efficacy. ${ }^{34}$

Long term follow-up after administration of human gene therapy suggests the safety of this approach. ${ }^{35}$ However, the integration of the carried gene into the host genome raises the potential for malignancies due to the disruption of critical host genes at the site of integration or activation of proto-oncogenes near the integration site.

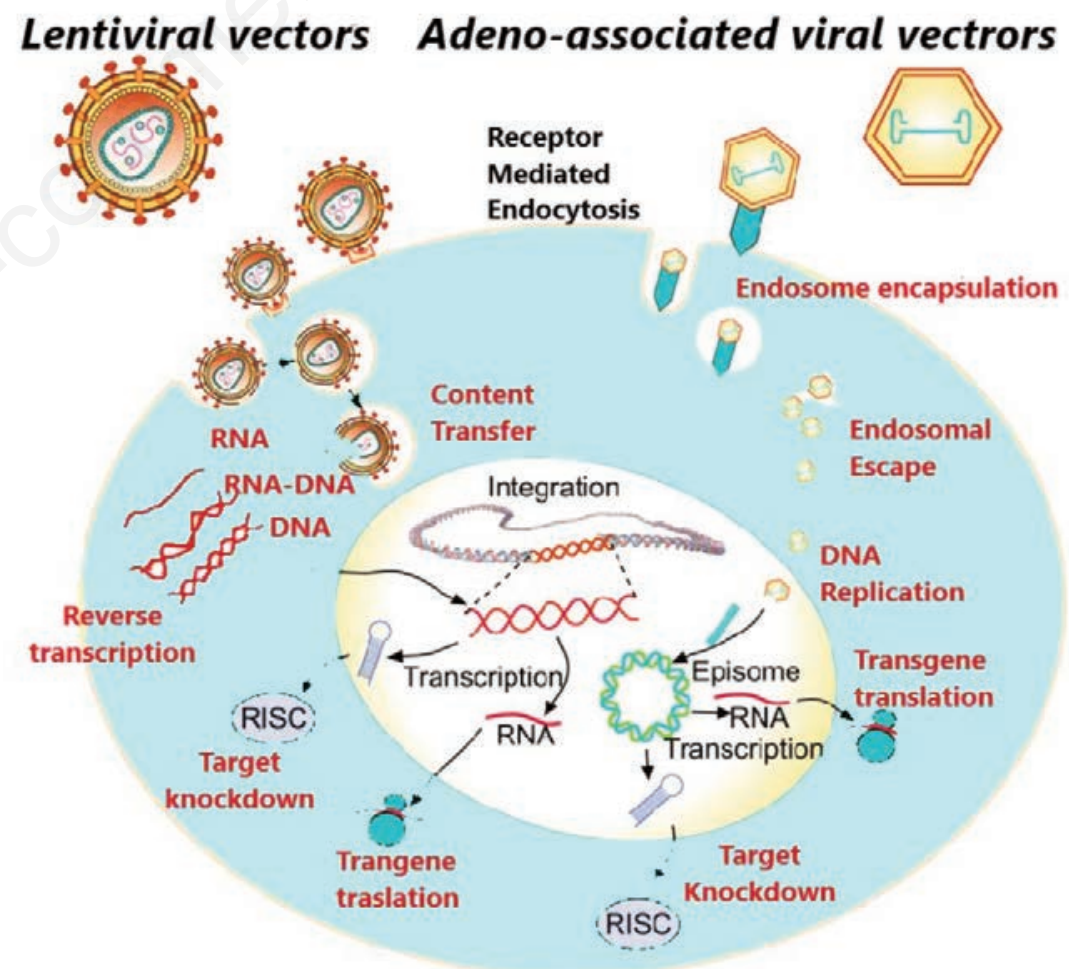

Figure 2. Lentiviral and adeno-associated viruses vector infection. Both vector systems are capable of transgene expression and endogenous gene knockdown. 
Recently, mRNA-based therapies emerged as an alternative for Fabry disease as studies in Fabry mice and non-human primates were reported. ${ }^{36}$ The use of transcripted mRNA carries the possibility to target specific amino acid modifications, bypassing the transcriptional process.

At the same time new gene editing techniques are in development, the most interesting being the molecular scissors system known as CRISP/Cas9 that, put into inactivated viral vectors, is then carried into the cells in order to edit their DNA. ${ }^{37}$ The same system could be used to create a human cell model of Fabry disease, useful as a target for testing new therapeutic strategies and medications.

\section{Conclusions}

Actually gene therapy in Fabry disease represents a promising alternative approach to enzyme replacement treatment. It consists of the introduction of a working copy of the gene encoding $\alpha$ galactosidase $\mathrm{A}$ in the patient's cells (fibroblasts, B lymphocytes, hepatocytes, hematopoietic cells) through lentivirus and adenovirus, showing encouraging results in some experimental animals with an increased enzymatic activity after few months of treatment.

Other approaches like chaperones and pseudoexon activation are under investigation too.

Among viral vectors, retrovirus (particularly Lentivirus) reported the best results in studies.

In conclusion, the aim of this emerging therapy is to create a more effective therapy for Anderson Fabry disease and a better outcome for these patients. However, there are still a lot of unanswered questions concerning gene therapy such as the correct timing of first administration to prevent or stop progression of organ damage, the possible influence of epigenetic and environmental factors and the lack of large randomized controlled trials.

\section{References}

1. Garman SC, Garboczi DN. The molecular defect leading to Fabry disease: structure of human alpha-galactosidase. J Mol Biol 2004;337:319-30.

2. Mehta A, Clarke JT, Giugliani R, et al. Natural course of Fabry disease: changing pattern of causes of death in FOS Fabry Outcome Survey. J Med Genet 2009;46:548-52.

3. Klingelhöfer D, Braun M, Seeger-
Zybok RK et al. Global research on Fabry's disease: Demands for a rare disease. Mol Genet Genomic Med 2020;00e1163.

4. Akhtar MM, Elliott PM. AndersonFabry disease in heart failure. Biophys Rev 2018;10:1107-19.

5. Eng CM, Fletcher J, Wilcox WR, et al. Fabry disease: Baseline medical characteristics of a cohort of 1765 males and females in the Fabry Registry. J Inherit Metab Dis 2007;30:184-92.

6. Kertesz B, Istvan E. Fabry Disease cardiomyopathy: from genes to clinical manifestations. Curr Pharm Biotechnol 2012;13:2477-84.

7. Limongelli G, Masarone D, Verrengia $\mathrm{M}$, et al. Diagnostic clues for the diagnosis of nonsarcomeric hypertrophic cardiomyopathy (phenocopies): amyloidosis, Fabry disease, and mitochondrial disease. J Cardiovasc Echogr 2018;28:120-3.

8. Augusto JB, Nordin S, Vijapurapu R, et al. myocardial edema, myocyte injury, and disease severity in Fabry disease. Circ Cardiovasc Imaging 2020;13: e010171.

9. Marian AJ. Challenges in the diagnosis of Anderson-Fabry Disease: a deceptively simple and yet complicated genetic disease. J Am Coll Cardiol 2016;68:1051-3.

10. Biegstraaten M, Hollak CE, Bakkers M et al. Small fiber neuropathy in Fabry disease. Mol Genet Metab 2012; 106:135-41.

11. Weidemann F, Niemann M, Breunig F, et al. Long-term effects of enzyme replacement therapy on Fabry cardiomyopathy: evidence for a better outcome with early treatment. Circulation 2009;119:524-9.

12. Mehta A, Hughes DA. Fabry disease. In: Adam MP, Ardinger HH, Pagon RA, et al. (eds.), GeneReviews ${ }^{\circledR}$. Seattle, WA: University of Washington; 1993.

13. Waldek S, Patel MR, Banikazemi M, et al. Life expectancy and cause of death in males and females with Fabry disease: findings from the Fabry Registry. Genet Med 2009;11:790-6.

14. Biegstraaten M, Arngrímsson R, Barbey F, et al. Recommendations for initiation and cessation of enzyme replacement therapy in patients with Fabry disease: the European Fabry Working Group consensus document. Orphanet J Rare Dis 2015;10:36.

15. Hoffmann B, Garcia de Lorenzo A, Mehta A, et al. Effects of enzyme replacement therapy on pain and health related quality of life in patients with Fabry disease: data from FOS (Fabry
Outcome Survey). J Med Genet 2005;42:247-52.

16. Weidemann F, Sanchez-Niño MD, Politei J, et al. Fibrosis: a key feature of Fabry disease with potential therapeutic implications. Orphanet J Rare Dis 2013;8:116.

17. Wilcox WR, Linthorst GE, Germain DP, et al. Anti-alphagalactosidase A antibody response to agalsidase beta treatment: data from the Fabry Registry. Mol Genet Metab 2012;105:443-9.

18. Lenders M, Brand E. Effects of enzyme replacement therapy and antidrug antibodies in patients with Fabry Disease. J Am Soc Nephrol 2018;29:2265-78.

19. $\mathrm{Xu} \mathrm{S}$, Lun Y, Brignol N, et al. Coformulation of a novel human $\alpha$ galactosidase a with the pharmacological chaperone AT1001 leads to improved substrate reduction in Fabry mice Mol Ther 2015;23:1169-81.

20. McCafferty E, Scott LJ. Migalastat: A Review in Fabry Disease. Drugs 2019;79:543-54.

21. Ortiz A, Germain DP, Desnick RJ. Fabry disease revisited: management and treatment recommendations for adult patients. Mol Gene Metab 2018;123:416-27.

22. Benjamin ER, Della Valle MC, Wu X, et al. The validation of pharmacogenetics for the identification of Fabry patients to be treated with migalastat. Genet Med 2017;19:430-8.

23. Warnock DG, Bichet DG, Holida M, et al. Oral migalastat $\mathrm{HCl}$ leads to greater systemic exposure and tissue levels of active alpha-galactosidase a in Fabry patients when co-administered with infused agalsidase. PLoS One 2015; 10:e0134341.

24. Cartegni L, Chew SL, Krainer AR. Listening to silence and understanding nonsense: exonic mutations that affect splicing nature reviews. Genetics 2002;3:285-98.

25. Palhais B, Dembic M, Sabaratnam R, et al. The prevalent deep intronic c. 639+919 G>A GLA mutation causes pseudoexon activation and Fabry disease by abolishing the binding of hnRNPA1 and hnRNP A2/B1 to a splicing silencer. Mol Genet Metab 2016;119:258-69.

26. Takahashi H, Hirai Y, Migita M, et al. Long-term systemic therapy of Fabry disease in a knockout mouse by adenoassociated virus-mediated muscledirected gene transfer. Proc Natl Acad Sci U S A 2002;99:13777-82.

27. Novo FJ, Gorecki DC, Goldspink G, et al. Gene transfer and expression of human alpha-galactosidase from mouse 
muscle in vitro and in vivo. Gene Ther 1997;4:488-92.

28. Jung SC, Han IP, Limaye A, et al. Adeno-associated viral vector mediated gene transfer results in long-term enzymatic and functional correction in multiple organs of Fabry mice. Proc Natl Acad Sci U S A 2001;98:2676-81.

29. Ziegler RJ, Cherry M, Barbon CM, et al. Correction of the biochemical and functional deficits in Fabry mice following AAV8-mediated hepatic expression of alpha-galactosidase A. Mol Ther 2007;15:492-500.

30. Kaissarian N, Kang J, Shu L, et al. Dissociation of globotriaosylceramide and impaired endothelial function in $\alpha$ -
galactosidase-A deficient EA.hy926 cells. Mol Genet Metab 2018;125:33844.

31. Sakuma T, Barry MA, Ikeda Y. Lentiviral vectors: basic to translational. Biochem J 2012;443:603-18.

32. Nagree MS, Scalia S, McKillop WM, Medin JA. An update on gene therapy for lysosomal storage disorders. Expert Opin Biol Ther 2019:1-16.

33. Sands MS, Davidson BL. Gene therapy for lysosomal storage diseases. Mol Ther 2006;13:839-49.

34. Nietupski JB, Hurlbut GD, Ziegler RJ, et al. Systemic administration of AAV8-a-galactosidase A induces humoral tolerance in nonhuman primates despite low hepatic expression. Mol Ther 2011;19:1999-2011.

35. DeRosa F, Smith L, Shen Y et al. Improved efficacy in a Fabry disease model using a systemic mRNA liver depot system as compared to enzyme replacement therapy. Mol Ther 2019;27:878-89.

36. Vargas JE, Chicaybam L, Stein RT et al. Retroviral vectors and transposons for stable gene therapy: advances, current challenges and perspectives. J Transl Med 2016;14:288.

37. Cong L, Ran FA, Cox D, et al. Multiplex genome engineering using CRISPR/Cas systems. Science 2013;339:819-23. 